\title{
Results of research of the eight- channel sensor for the defectoscopy of railway rails
}

Vitalij Nichoha, Volodymyr Storozh, Yurij Matiieshyn

Vitalij Nichoha, Volodymyr Storozh, Yurij Matiieshyn, "Results of research of the eight-channel sensor for the defectoscopy of railway rails," Proc. SPIE 11442, Radioelectronic Systems Conference 2019, 114421A (11 February 2020); doi: $10.1117 / 12.2565748$

SPIE. Event: Radioelectronic Systems Conference 2019, 2019, Jachranka, Poland 


\title{
Results of research of the eight-channel sensor for the defectoscopy of railway rails
}

\author{
Vitalij Nichoha*, Volodymyr Storozh ${ }^{\dagger}$, Yurij Matiieshyn * \\ Lviv Polytechnic National University, 12 Bandery St., 79013 Lviv, Ukraine
}

\begin{abstract}
The paper presents results of the development and experimental study of an eight-channel search sensor for railway rails defectoscopy. The experimental sample of the search sensor is implemented on the basis of Hall sensors and is designed to record the changes of the longitudinal component of the scattering magnetic field of the structural elements of the track and defects. The choice, for the registration of defectoscopic signals, changes in the longitudinal component of the scattering magnetic field is due to the fact that the same component of the field is registered by regular singlechannel induction search sensors of the carriages-defectoscopes. The investigated search sensor contained eight Hall sensors SS49E, which were placed at intervals of approximately $1 \mathrm{~cm}$ in a row on a special board, which was fixed to the search ski of the carriage-defectoscope. Thus, an eight-channel search sensor covered the entire surface of the rail head. Defectoscopic information from eight Hall sensors was synchronously recorded on a personal computer using a multi-channel data acquisition system with a sampling rate of $20 \mathrm{kHz}$ and a bit resolution of 12-bit of analog-to-digital converter. During the experimental researches on the carriage-defectoscope, with departure to the track section of the Lviv railway, it was found that the eight-channel search sensor registers all structural elements of the railway track, including welded rail joints. The adequacy of the records was proved by numerically differentiating the signal from the bolt rail joint. As a result of such verification, a signal was obtained, the shape of which corresponds to the signal of the induction sensor from the bolt rail joint, which is common to carriage-defectoscope operators. The signals of the adjacent channels are practically coincided. The worst case correlation coefficients were not less than 0,95 .
\end{abstract}

Keywords: search sensor, defectoscopic signal, rail.

\section{INTRODUCTION}

Due to the high congestion of the railway lines, the urgent task is to ensure the speed diagnostics of the railway track without reducing their throughput. To do this, various diagnostic tools based on different physical principles are used. The most common of these are ultrasonic and magnetic.

Ultrasonic methods provide diagnostics of the entire transverse section of the rail, which allows estimating the sizes and positions of defects [1]. This ensures their sustainable development, refinement and implementation. However, they are sensitive to weather conditions and pollution of the rail track, requiring constant acoustic contact between the sensor and the rail, which causes unsatisfactory detection of surface defects. The loss of acoustic contact often occurs with sharp turns of the rail track.

Unlike ultrasonic, magnetic methods [1-4] perfectly diagnose only the surface defects of the rail head and do not give information about their transverse location. Their advantage is the insensitivity to weather conditions and pollution of the track, the ability to register the structural elements of the track, which facilitates the spatial anchoring and determining the location of the carriage-defectoscope. They lack the loss of information at sharp turns, which is important for diagnostics, in particular, in mountainous regions of the country.

Therefore, the work is devoted to increasing the information content of the magnetic channels of mobile means of rail defectoscopy using multichannel sensors with the possibility of visualization of the investigated section of the rail.

\footnotetext{
*nich@org.lviv.net

†volodymyr.h.storozh@1pnu.ua

*yurii.m.matiieshyn@1pnu.ua
}

Radioelectronic Systems Conference 2019, edited by Piotr Kaniewski, Jan Matuszewski, Proc. of SPIE Vol. 11442, 114421A · C 2020 SPIE · CCC code: 0277-786X/20/\$21 · doi: 10.1117/12.2565748 


\section{RESULTS OF THE EIGHT-CHANNEL SENSOR DEVELOPMENT}

In this paper the issues of the development and research of an eight-channel sensor for the railway rails defectoscopy are presented. The investigated search sensor contain eight Hall sensors SS49E, which were placed at intervals of approximately $1 \mathrm{~cm}$ in a row on a special board and focused on the registration of the longitudinal, relative to the axis of the rail, component of the scattering magnetic field of regular track elements and defects (Fig. 1). The sensor is mounted on the search ski of the carriage-defectoscope, providing scanning of entire surface of the rail head. In contrast to the currently used single-channel induction sensors, the developed eight-channel sensor based on Hall sensors can receive not only a series of signals about the fact of a defect, but also about the spatial location of possible defects.

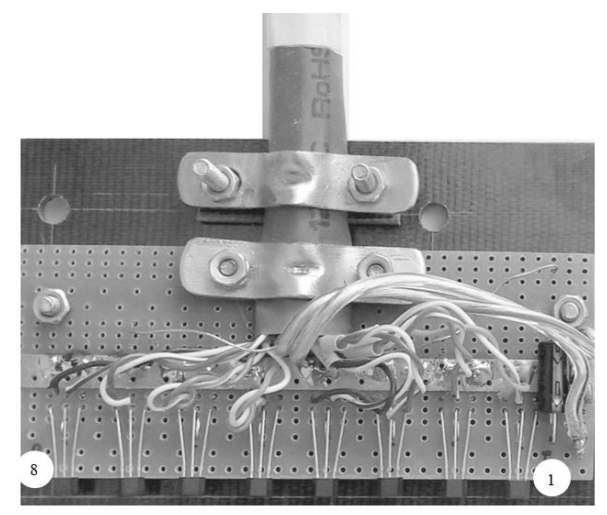

Figure 1. An experimental model of an eight-channel sensor.

Defectoscopic information from eight independent Hall sensors is synchronously recorded on a personal computer using a multi-channel data acquisition system, forming eight independent information channels for each rail. Sampling rate is $20 \mathrm{kHz}$ and resolution of analog-to-digital converter - 12-bit.

\section{RESULTS OF NATURAL TESTS}

\subsection{The results of the recording of defectoscopic signals}

The performance of an eight-channel search sensor based on Hall sensors with a data acquisition system was tested in process of section of the Lviv railway track inspection on the carriage-defectoscope, taking into account the production capabilities of the Lviv Diagnostic Center structural unit. The main purpose of this phase of research was to check the adequacy of the recording of defectoscopic information. Search ski with the eight-channel sensor under test was installed in place of a regular induction sensor of the carriage-defectoscope sensor near the rear, in the course of the carriage movement, pole of the electromagnet of magnetizing system (Fig. 2).

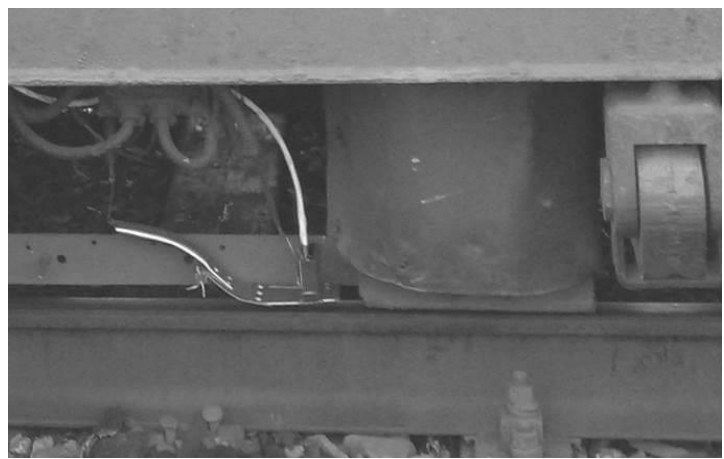

a

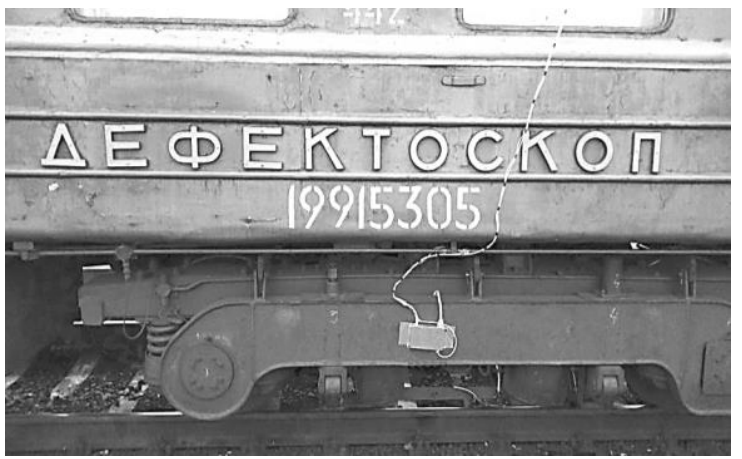

$\mathrm{b}$

Figure 2. Eight-channel sensor (a), with multichannel data acquisition system (b) on the inductor cart of the carriage-defectoscope. 
On Figure 3 are shown eight overlapping signals from the same bolt rail joint, which are synchronously recorded with an eight-channel sensor based on Hall sensors during the track inspection. Fluctuations of insignificant amplitude correspond to the signal from the sleeper overlays, the positive long pulse - the signal from the rail, and the short pulse of negative polarity - the signal from the joint.

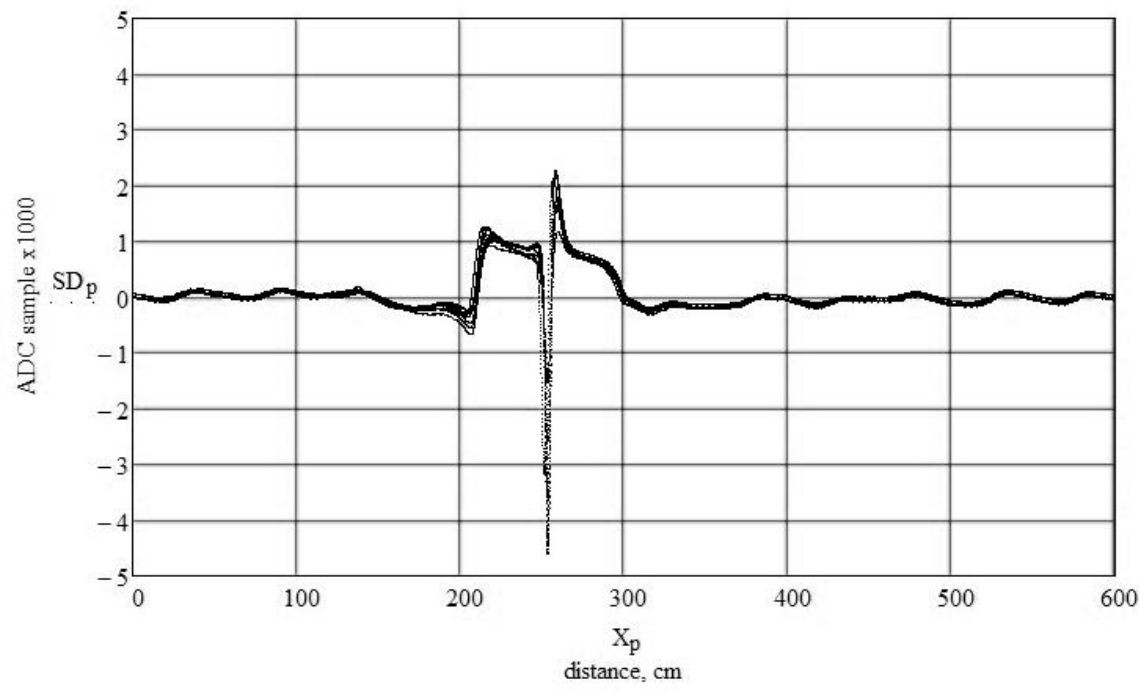

Figure 3. Eight superimposed on each other signals from the bolt rail joint recorded by an eight-channel sensor.

It is possible to obtain a signal that corresponds to the signal of a standard induction sensor of a carriage-defectoscope, which is familiar to defectoscope operators, by means of numerical differentiation:

$$
S I_{p}=-\frac{S H_{p}-S H_{p-a}}{X_{p}-X_{p-a}}
$$

where $\mathrm{SH}$ - signal obtained by the Hall sensors;

SI - signal equivalent to the signal of an induction sensor of a carriage-defectoscope, obtained by numerical differentiation of the signal $S H$;

$p$ - element number of the array;

$a-$ a step of numerical differentiation.

The result of the numerical differentiation of the signal from the bolt rail joint obtained with the sensor under study (Fig. 3) is presented in Figure 4.

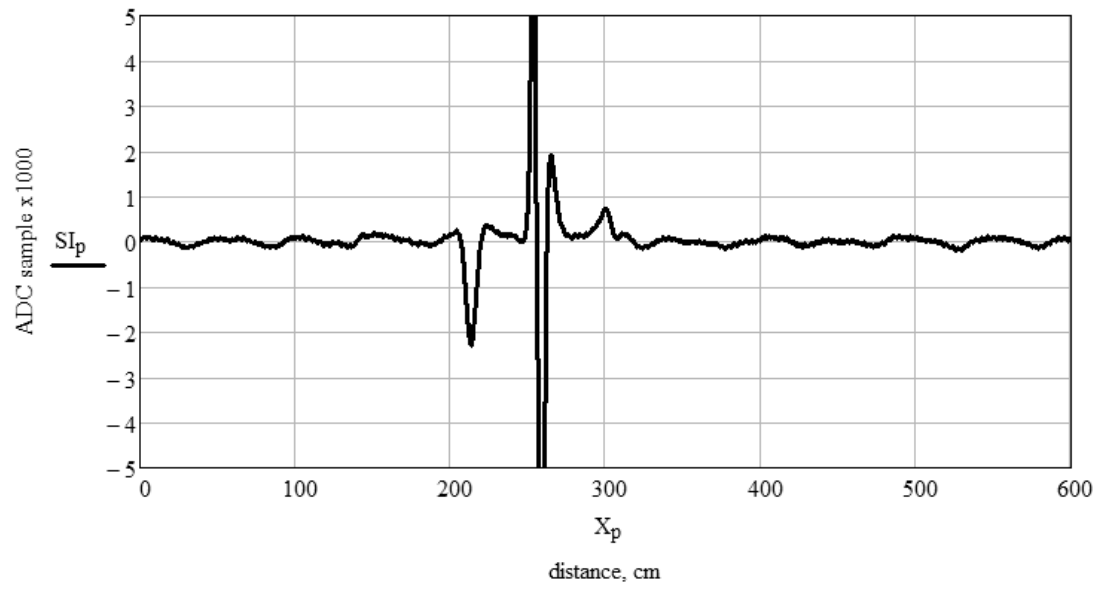

Figure 4. Signal from bolt rail joint obtained by numerical differentiation. 
The received signal form is a typical signal form from a bolt rail joint, which can be obtained with an induction sensor [2], which confirms the adequate registration of defectoscopic signals.

When analyzing the signals from the regular elements of the track, it is found that the signals of the adjacent channels of the eight-channel sensor are almost identical. First of all, it concerns the rail joints. However, during the study it was found that the signals from some of the structural elements in each sensor channel are different. Obviously, this is due to their design features, first of all, asymmetry with respect to the axis of the rail. For example, in Figure 5 the results of recording signals of the eight-channel encoder from the structural elements of the track are present. The signals are inverted in the process of preparation for the construction of the image of the surface of the rail head, which will be discussed below.

The first signal is from a structural element that is asymmetrical about the axis of the rail. The figure shows that since the third channel signals are differ from each other. The second - signal from the bolt rail joint. The signals forms in the measuring channels are almost identical. Some decrease in the amplitude of the impulse from the gap between the rails is caused by a slight misalignment of the search ski on which the eight-channel sensor was installed, since the signal amplitude from the rail pads of this joint is practically the same in all channels. It was not possible to eliminate the ski misalignment during the movement of the carriage-defectoscope.

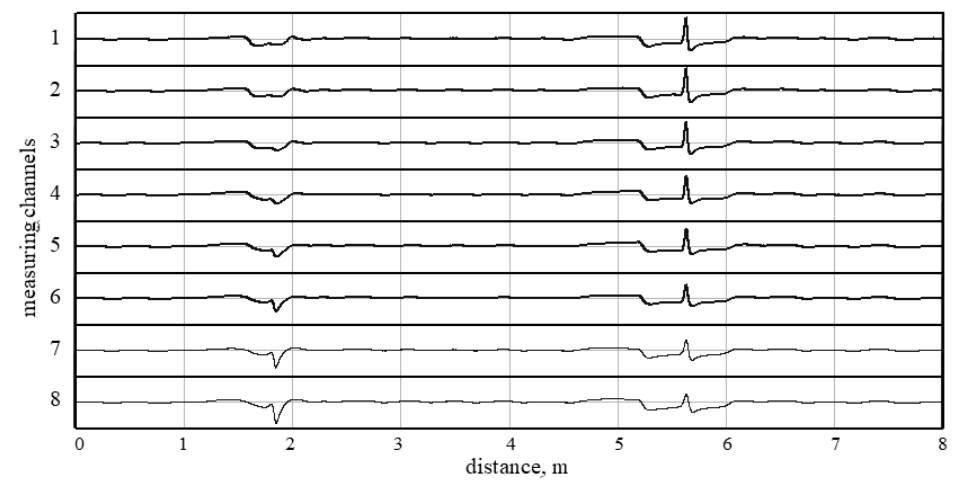

Figure 5. The signals of the eight-channel sensor from the structural elements of the track.

To determine the degree of similarity of signals in individual channels, the corresponding correlation coefficients were calculated. Since the individual Hall sensors of the eight-channel sensor are located strictly across the rail, the $S H$ signals obtained with the help of the developed eight-channel sensor are combined in time, which simplifies the procedure of calculating the correlation coefficients:

$$
C_{n}=\frac{\sum_{p}\left(S H_{1, p}-\overline{S H_{1, p}}\right)\left(S H_{n, p}-\overline{S H_{n, p}}\right)}{\sqrt{\sum_{p}\left(S H_{1, p}-\overline{S H_{1, p}}\right)^{2} \cdot \sum_{p}\left(S H_{n, p}-\overline{S H_{n, p}}\right)^{2}}},
$$

where $n=1 . .8$ - serial number of the signal of the eight-channel sensor based on Hall sensors; $\mathrm{p}-$ serial number of readout of the signal.

In Figure 6 the values of correlation coefficients that were calculated between the signals (Fig. 5) of the first sensor and all subsequent ones are presented. 


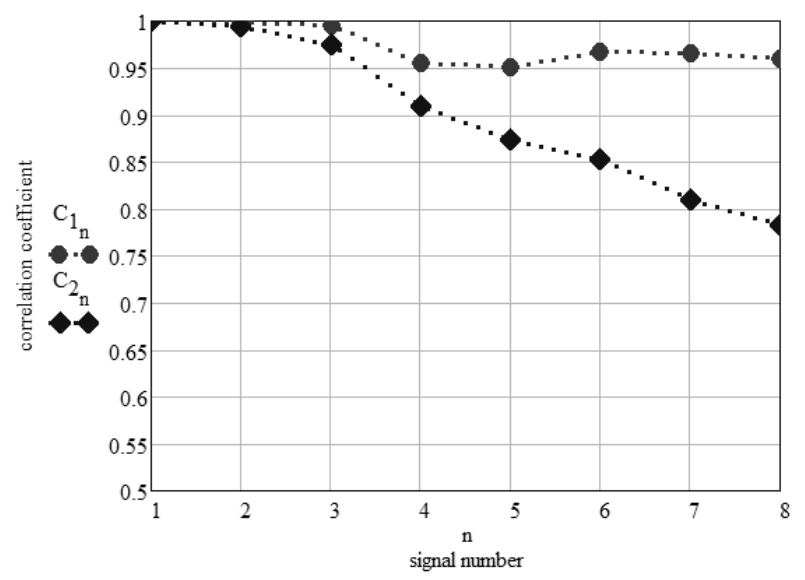

Figure 6. Correlation coefficients between the signal of the first channel of the eight-channel sensor and the signals of the remaining channels for different structural elements of the track.

Notation in the Figure 6: $C_{1}$ - correlation coefficients between the signals of the individual sensors for the bolt joint, $C_{2}$ -correlation coefficients for a structural element that is asymmetric about the axis of the rail. As can be seen from the figure, for the bolt rail joint correlation coefficients do not fall below 0.95 . At the same time, the asymmetric structural element has a monotonic decrease in the correlation coefficients. Such distinguishing of signals in adjacent channels will allow, in the future, detecting the position of surface defects in the rail head.

\subsection{Visualization of defectoscopic signals}

The presence of eight synchronous signals allows constructing an image of the entire surface of the rail head in the study area [5]. In the work, this is realized by forming an image whose brightness is proportional to the magnitude of the magnetic scattering field. In Figure 7 below the multichannel signal, a visualization of the rail section under study is given.
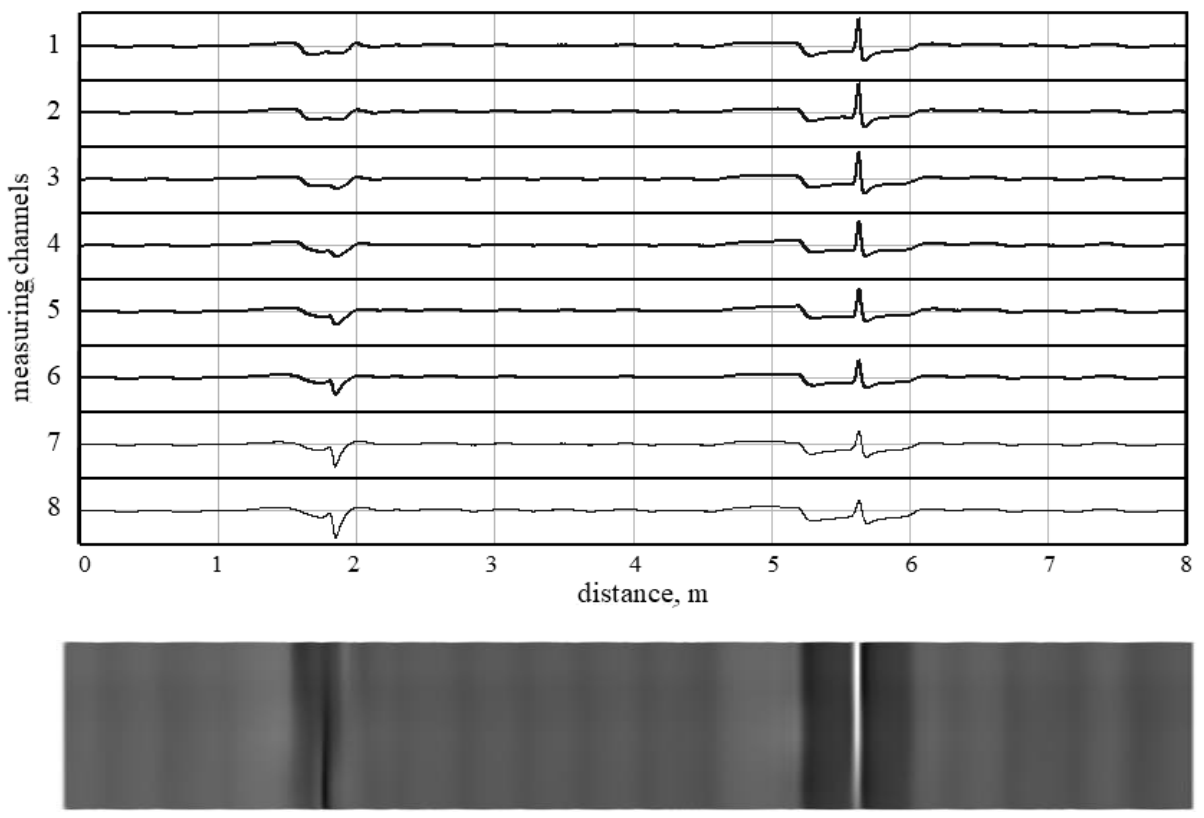

Figure 7. Fragment of recording of the eight-channel signal from the structural elements of the track and its visualization. 
Vertical dark stripes corresponding to the sleeper overlays are traced throughout the image. It can be seen that the first structural element is asymmetrical with respect to the axis of the rail, and its dark tint indicates that it is a ferromagnetic object. The second structural element - bolt rail joint. The light line figure corresponds to the gap between the adjacent rails. The dark wide strip corresponds to the rail overlay.

\section{CONCLUSIONS}

Using of the proposed eight-channel sensor, in addition to detecting signals from the structural elements of the railway track and possible defects, allows to visualize the investigated section of the rail by forming an image whose brightness is proportional to the magnitude of the scattering magnetic field of this section. This gives additional information about the degree of transverse homogeneity of these elements, will allow more clearly to identify the structural elements of the track, to determine the approximate dimensions of defects and to locate them in the transverse section of the rail head, with respect to its working or non-working sides. The latter can be useful in determining the degree of risk of found defects.

\section{REFERENCES}

[1] Y. M. Matiieshyn, V. O. Nichoha, V. I. Shkliarskyi, V. G. Storozh and L. V. Vashchyshyn: "The Research of modern methods and means of high-speed diagnostics of railway rails defects (Review)", Informacijnokerujuchi systemy na zaliznychnomu transporti, 2018, № 3(130), P. 8-22.

[2] G.A. Antipov, N.A. Belousov and A.A. Markov: "Comparative analysis of signals of magnetic channels of mobile means of rail defectoscopy", Multipurpose radioelectronic complexes, St. Petersburg, Radioavionica, 2006. - Available on: http://www.radioavionica.ru/about/events/stati-razrabotchikov-oao-radioavionika/298/.

[3] Markov A.A. Application of correlation analysis for weld joint inspection by magnetic flux leakage method / A.A. Markov, G.A. Antipov // V mire NK. - 2015. - 2(68). - P. 74-77.

[4] Markov A.A. Possibilities of the magnetic flux leakage method of rail defectoscopy / A.A. Markov, G.A. Antipov // Control. Diagnostics. - 2016. - № 6. - P. 36-45.

[5] Yong Li, John Wilson and Gui Yun Tian: "Experiment and simulation study of 3D magnetic field sensing for magnetic flux leakage defect characterisation", NDT \& E International, Elsever LTD, 2007, Vol. 40(2), P. 179-184. - Available on: http://dx.doi.org/10.1016/j.ndteint.2006.08.002. 